\title{
TIMP-2 modulates cancer cell transcriptional profile and enhances E-cadherin/beta-catenin complex expression in A549 lung cancer cells
}

\author{
Dimitra Bourboulia1, HuiYing Han ${ }^{1}$, Sandra Jensen-Taubman ${ }^{1, *}$, Noah Gavil ${ }^{1,2, *}$, \\ Biju Isaac ${ }^{1,3}$, Beiyang Wei, Len Neckers ${ }^{4}$ and William G. Stetler-Stevenson ${ }^{1}$ \\ ${ }^{1}$ Radiation Oncology Branch, Center for Cancer Research, National Cancer Institute, Advanced Technology Center, 8717 \\ Grovemont Circle, Bethesda, MD, USA \\ 2 Bowdoin College, Brunswick, ME, USA \\ ${ }^{3}$ Center for Computational Science, University of Miami, Miami, FL, USA \\ ${ }^{4}$ Urologic Oncology Branch, Center for Cancer Research, National Cancer Institute, Bethesda, MD, USA \\ * Denotes equal contribution \\ Correspondence to: Len Neckers, email: neckers@nih.gov
}

William G. Stetler-Stevenson, email: sstevenw@mail.nih.gov

Keywords: TIMP-2, microarray analysis, E-cadherin, cell adhesion, tumor growth inhibition

Received: December 27, 2012, Accepted: January 26, 2013, $\quad$ Published: January 27, 2013

Copyright: ( Bourboulia et al. This is an open-access article distributed under the terms of the Creative Commons Attribution License, which permits unrestricted use, distribution, and reproduction in any medium, provided the original author and source are credited.

\section{ABSTRACT:}

Tissue Inhibitor of Metalloproteinase 2 (TIMP-2) plays an essential role in regulating matrix remodeling, cell growth, differentiation, angiogenesis and apoptosis in vitro and in vivo. We have recently shown that TIMP-2-mediated inhibition of tumor growth is independent of matrix metalloproteinase-mediated mechanisms, and is a consequence of modulating both the tumor cells and the tumor microenvironment. In the current study we aim to identify the molecular pathways associated with these effects. We analyzed the transcriptional profile of the human lung cancer cell line A549 upon overexpression of TIMP-2 and Ala+TIMP-2 (mutant that does not inhibit MMP activity), and we found changes in gene expression predominantly related to decreased tumor development and metastasis. Increased E-cadherin expression in response to both TIMP-2 and Ala+TIMP-2 expression was confirmed by real time quantitative RT-PCR and immunoblotting. A549 cells treated with epidermal growth factor (EGF) displayed loss of cobblestone morphology and cell-cell contact, while cells overexpressing TIMP-2 or Ala+TIMP-2 were resistant to EGF-induced morphological changes. Moreover, exogenous treatment with recombinant Ala+TIMP-2 blocked EGF induced down-regulation of E-cadherin. In vivo, immunohistochemistry of A549 xenografts expressing either TIMP-2 or Ala+TIMP-2 demonstrated increased E-cadherin protein levels. More importantly, transcriptional profile analysis of tumor tissue revealed critical pathways associated with effects on tumor-host interaction and inhibition of tumor growth. In conclusion, we show that TIMP-2 promotes an anti-tumoral transcriptional profile in vitro and in vivo, including upregulation of E-cadherin, in A549 lung cancer cells.

\section{INTRODUCTION}

TIMPs are a family of proteins that naturally inhibit the activity of matrix metalloproteinases (MMPs). Uncontrolled MMP proteolysis promotes extracellular matrix degradation, tumor cell invasion and increased risk for cancer cell metastasis [1-3]. TIMP-2 is a unique TIMP family member, in that it both inhibits active MMP-2 as well as activates pro-MMP-2 in the presence of a membrane tethered MMP, MMP14/MT1-MMP $[4,5]$. TIMP-2 functions as an endogenous inhibitor of both angiogenesis and tumor growth, effects that may 
be dependent or independent of MMP inhibition [612]. Known mechanisms associated with inhibition of endothelial cell proliferation and migration in vitro and angiogenesis in vivo include: a) TIMP-2 binding to integrin $\alpha_{3} \beta_{1}$ receptor and activation of $\mathrm{SH} 2$-containing protein tyrosine phosphatase-1 (SHP-1), which suppresses the activity of receptor tyrosine kinases VEGFR2 and FGFR1 upon growth factor stimulation (VEGF-A or FGF2); and b) C-terminus TIMP-2 loop 6 binding to insulin-like growth factor receptor I (IGF-IR) to disrupt downstream mitogenic signaling through AKT hypo-phosphorylation [9, 11, 13]. Major changes within downstream signal transduction pathways involve induction of cell cycle arrest with de novo synthesis of tumor suppressor gene $\mathrm{p} 27^{\mathrm{kip} 1}$, retinoblastoma protein $(\mathrm{pRb})$ hypo-phoshorylation, and up-regulation of the MMP inhibitor, reversioninducing-cystein-rich protein with Kazal motif (RECK), via modification of paxillin phosphorylation and Rap1 upregulation [14-18].

More recently, forced expression of TIMP-2 in A549 human lung cancer cells was performed to address whether TIMP-2 overexpression directly influences tumor angiogenesis and/or tumor cell behavior. Indeed, tumor cell migration and invasion were inhibited in vitro, whereas in vivo tumor growth inhibition was achieved through TIMP-2 mediated interaction with the tumor microenvironment, angiogenesis inhibition and induction of tumor cell apoptosis [12]. The use of Ala+TIMP-2 in similar experiments shows that mechanistically, inhibition of MMP activity does not entirely explain all TIMP-2 functions [19].

Therefore, it is apparent that TIMP-2 plays a broader role both in endothelial cell physiology and in cancer development [20]. In an attempt to understand how TIMP2 regulates angiogenesis and tumor growth inhibition, we performed human cDNA microarray analysis and compared the differential gene expression profiles of A549 tumor cells overexpressing TIMP-2 or Ala+TIMP-2 with that of stably transfected Empty Vector control cells (EV) in vitro and in vivo. We found that the TIMP2 transcriptional signature in A549 cells is associated with modulation of genes that inhibit endothelial cell proliferation, angiogenesis, lung cancer development and metastasis. Specifically, we show that the tumor suppressor gene E-cadherin is up-regulated by TIMP-2 overexpression in vitro and in vivo, contributing to the maintenance of cell-cell adhesion that may also contribute to inhibition of tumor growth.

\section{RESULTS AND DISCUSSION}

\section{Differentially expressed gene profiles in TIMP-2 and Ala+TIMP-2 transfected A549 cell lines.}

To identify pathways resulting from TIMP-2 mediated effects on tumor cells and the microenvironment, we performed microarray analysis on TIMP-2 and Ala+TIMP-2 overexpressing A549 cells, previously shown to display reduced migration and invasion in vitro, and tumor growth and angiogenesis in vivo [12, 21]. The microarray data can be found at the link: http:// www.ncbi.nlm.nih.gov/geo/query/acc.cgi?token=nxm zxmkaqgisipu\&acc $=$ GSE38408. Analysis of variance (ANOVA) of the data (see methods) identifies a subset of 2480 differentially expressed (DE) genes across the three A549 experimental groups: EV, TIMP-2 and Ala+TIMP-2 (Fig. 1A). Hierarchical clustering of DE genes using the 'Pearson centered' distance similarity method and 'average' linkage rule suggests that specific clusters of enriched gene functions belong exclusively to TIMP-2 (clusters B \& F) or Ala+TIMP-2 (clusters D \& E) groups, whereas other clusters (A \& C) were common to both groups (Fig 1A). This analysis reveals several findings: the TIMP-2/Ala+TIMP-2 commonly expressed genes (clusters A \& C) identify an MMP-independent function of TIMP-2, whereas genes that change predominantly in TIMP-2 overexpressing cells (clusters B \& F) may a result of MMP inhibition. Finally, genes affected solely in the Ala+TIMP-2 group (clusters D \& E) may depend on MMP activation.

Common AC genes in TIMP-2 or Ala+TIMP-2 samples were primarily associated with cellular movement, death, growth, signaling and metabolism (Supplementary Fig. 1A). Comprehensive analysis revealed that the most up- and down-regulated genes, in either TIMP-2 or Ala+TIMP-2 samples, were related to decreased cancer development and metastasis (Fig. 1B). More specifically, the secreted protein EFEMP1 (up-regulated) was recently shown to suppress malignant glioma growth [22], and $D L C-1$ (up-regulated) is a well-known tumor suppressor gene that regulates cell proliferation and migration [23]. On the other hand, CEACAM6 (down-regulated) is overexpressed in a number of epithelial malignancies, particularly in invasive pancreatic cancer [24], and NTS (down-regulated) is shown to promote cancer cell growth [25]. In addition, decreased gene function associated with lung cancer development was shown in both TIMP-2 and Ala+TIMP-2 samples: IGFBP3 and $C D H 1$ were upregulated, and $S 100 P$ and $P T G S 2$ were down-regulated [26-30]. These data indicate that TIMP-2 overexpression transcriptionally regulates tumor cell development and growth, independent of MMP inhibition, although further work is needed to identify specific mechanisms. TIMP-2 


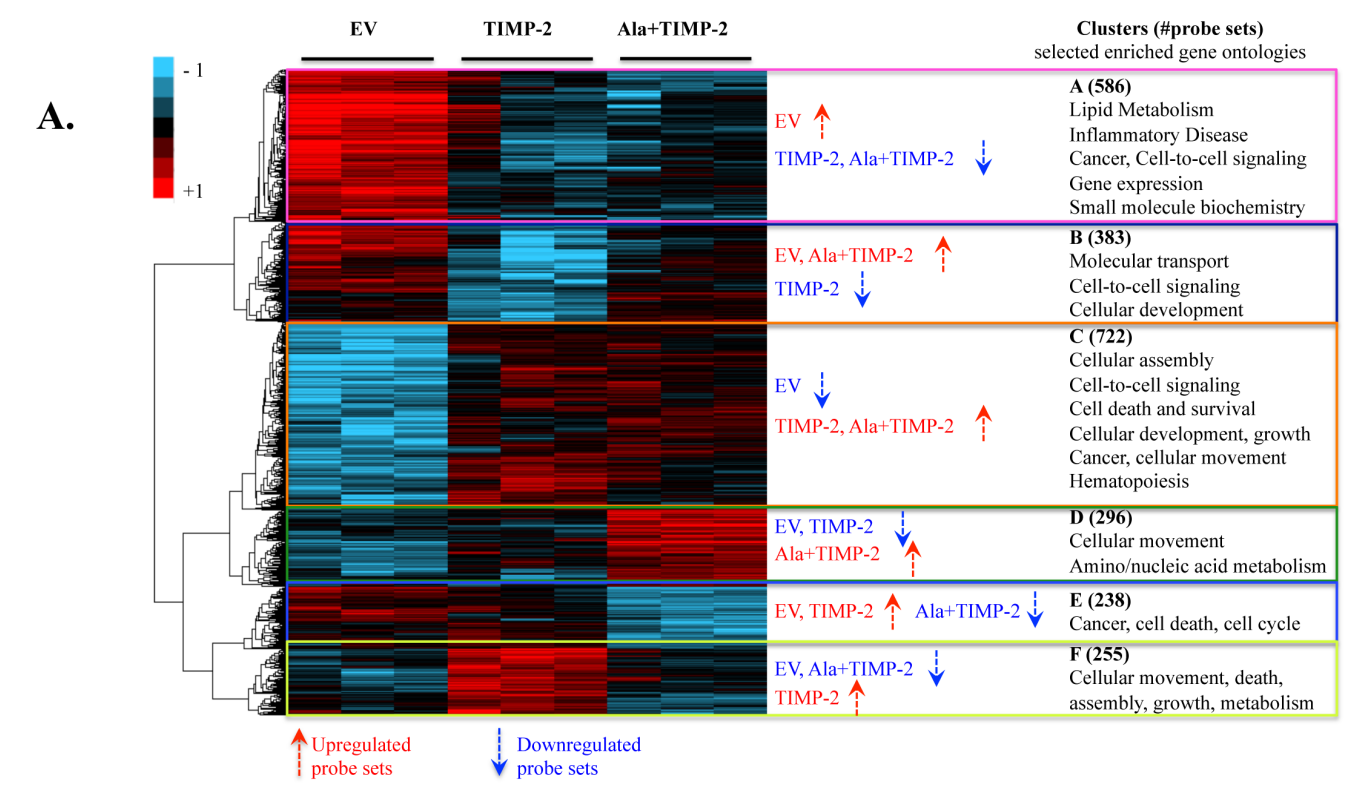

B.

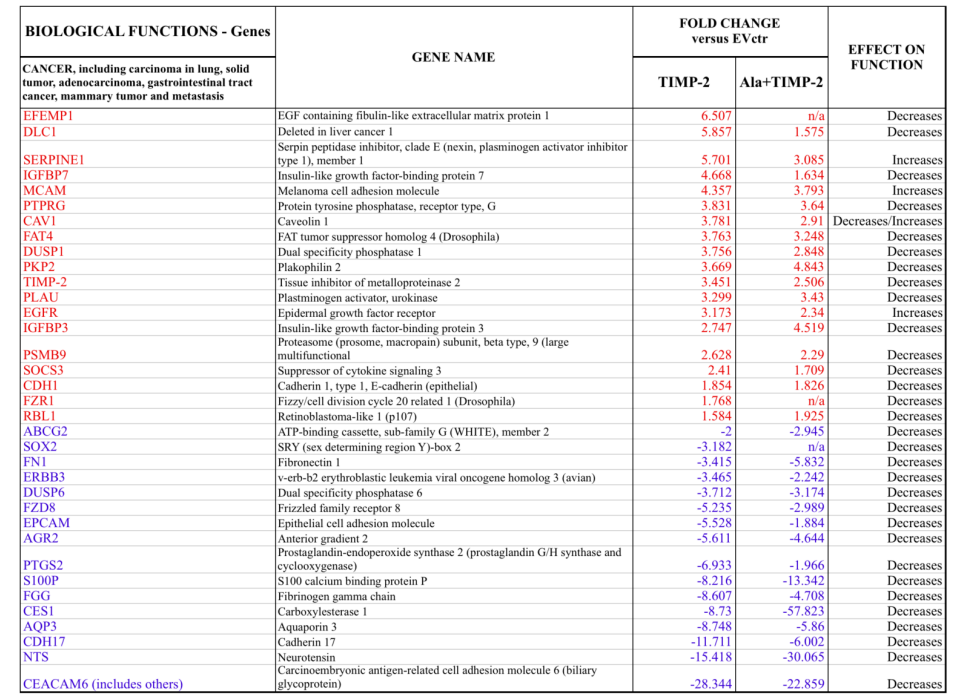

C.

\begin{tabular}{|c|c|c|c|}
\hline \begin{tabular}{|l} 
BIOLOGICAL FUNCTIONS - Genes \\
\end{tabular} & \multirow[b]{2}{*}{ GENE NAME } & $\begin{array}{l}\text { FOLD } \\
\text { CHANGE }\end{array}$ & \multirow{2}{*}{$\begin{array}{l}\text { EFFECT ON } \\
\text { FUNCTION }\end{array}$} \\
\hline $\begin{array}{l}\text { ANGIOGENESIS, including tumor angiogenesis, } \\
\text { proliferation and development of endothelial cells } \\
\text { and vascularization }\end{array}$ & & TIMP-2 vs EV & \\
\hline CYR61 & Cystein-rich 61 & 3.971 & Increases \\
\hline $\mid \begin{array}{l}\text { TAV1 } \\
\text { TIMP2 (includes EG:21858) }\end{array}$ & $\begin{array}{l}\text { Caveolin } 1 \\
\text { Tissue Inhibitor of Metalloproteinase } 2\end{array}$ & 3.7811 & $\begin{array}{c}\text { Decreases } \\
\text { Decreases }\end{array}$ \\
\hline COL4A1 & 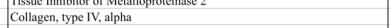 & 3.45 & $\begin{array}{l}\text { Decreases } \\
\text { Decreases }\end{array}$ \\
\hline COL4A2 & Collagen, type IV, alpha 2 & 2.992 & Decreases \\
\hline VLDLR & $\begin{array}{l}\text { Very low density lipoprotecin reccptor } \\
\text { Semald domain }\end{array}$ & & \\
\hline SEMA3A & $\begin{array}{l}\text { Semala domain, iminunogobu } \\
\text { (semaphorin) } 3 \mathrm{~A}\end{array}$ & 2.588 & Decreases \\
\hline STAT1 & Signal transstucer and activator of transcription $1,91 \mathrm{kDa}$ & $\begin{array}{r}2.468 \\
2.391\end{array}$ & Decreases \\
\hline & $\begin{array}{l}\text { Plasmingogen activator, tissue } \\
\text { Caveolin2 }\end{array}$ & $\begin{array}{r}2.391 \\
2.38\end{array}$ & \\
\hline KLF & Kruppel-like factor 5 (intestinal) & $\begin{array}{r}-2.923 \\
-1.923\end{array}$ & $\begin{array}{l}\text { Decieases } \\
\text { Decreases }\end{array}$ \\
\hline EDIL3 & EGF-like repeats and discoidin I-like domains 3 & -2.811 & Decreases \\
\hline ANGPT1 & Angiopoietin I & -2.84 & Decreases \\
\hline \begin{tabular}{|l} 
AGIR \\
ANG
\end{tabular} & $\begin{array}{l}\text { Angiotensini II receptor, type I } \\
\text { Angiogenen, ribonuclease, RNase A family, } 5\end{array}$ & $\begin{array}{l}-3.027 \\
-3.088\end{array}$ & $\begin{array}{c}\text { Decreases } \\
\text { Decreases }\end{array}$ \\
\hline & Fib & -3.415 & Decreases \\
\hline TNFSF 10 & Tumor necrosis factor (ligand) superfamily, member 10 & -4.167 & Decreases \\
\hline PTGS2 & 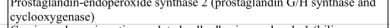 & -6.933 & Decreases \\
\hline CEACAMl in & $\begin{array}{l}\text { Carcinoembrry } \\
\text { glycoprotetin) }\end{array}$ & -28.344 & Decreases \\
\hline
\end{tabular}

Figure 1: TIMP-2 is a transcriptional regulator of human lung cancer A549 cells in vitro. (A) Clustered heat map diagram of microarray data for EV, TIMP-2 and Ala+TIMP-2 overexpressing A549 cells (three replicates). 2480 probe sets determined to significantly change across the three groups with false discovery rate (FDR) $<10$ are shown in the heat map. The key on the top left assigns heat map colors to the absolute gene expression value on a $\log _{2}$ scale. The most significant Gene Ontology (GO) categories, with the number of probe sets in parentheses, identifying enriched biological processes for each cluster across A549 TIMP-2 and Ala+TIMP-2 cells are shown next to the heat map. Bivariate comparisons of TIMP-2 (B and C) or Ala+TIMP-2 (B) with EV generated lists of functions unique for each comparison. Selected functions (cancer, B and angiogenesis, C) and transcriptional regulators with gene lists and predicted effect on function are shown. The most highly up-regulated (red) and down-regulated (blue) transcripts are presented with at least 1.5 -fold change versus EV control. 
also exhibited significant changes related to angiogenesis inhibition and endothelial cell proliferation, including up-regulation of $C A V 1, C O L 1 A$ and $S E M A 3 A$, and downregulation of CEACAM1, PTGS2, and FN1 (Fig. 1C) [31-37]. Taken together, these data suggest that TIMP-2 overexpression in tumor cells could inhibit angiogenesis through paracrine effects on tumor endothelium, while
A.
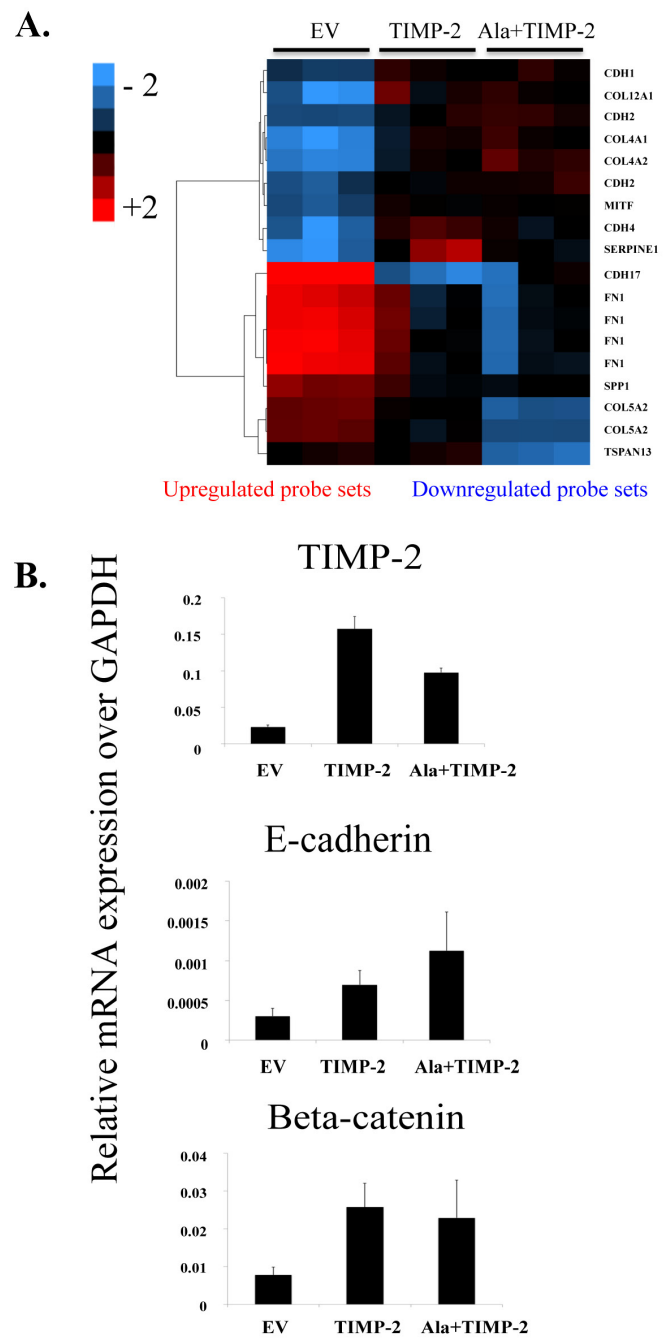

C.

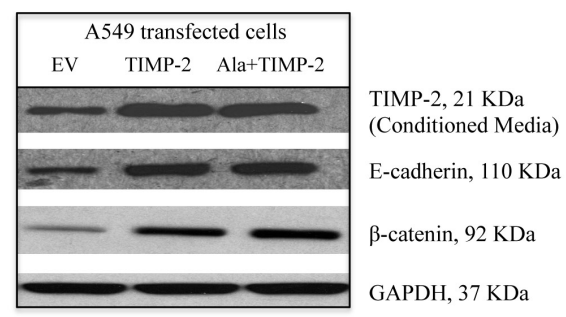

decreasing tumor growth directly through regulation of the tumor cell transcriptome. In addition, since Ala+TIMP-2 overexpression showed similar findings, our data may partially explain the MMP-independent activity exhibited by TIMP-2 (illustrated in previous and recent literature [9, $10,12,14,18,38-41])$.

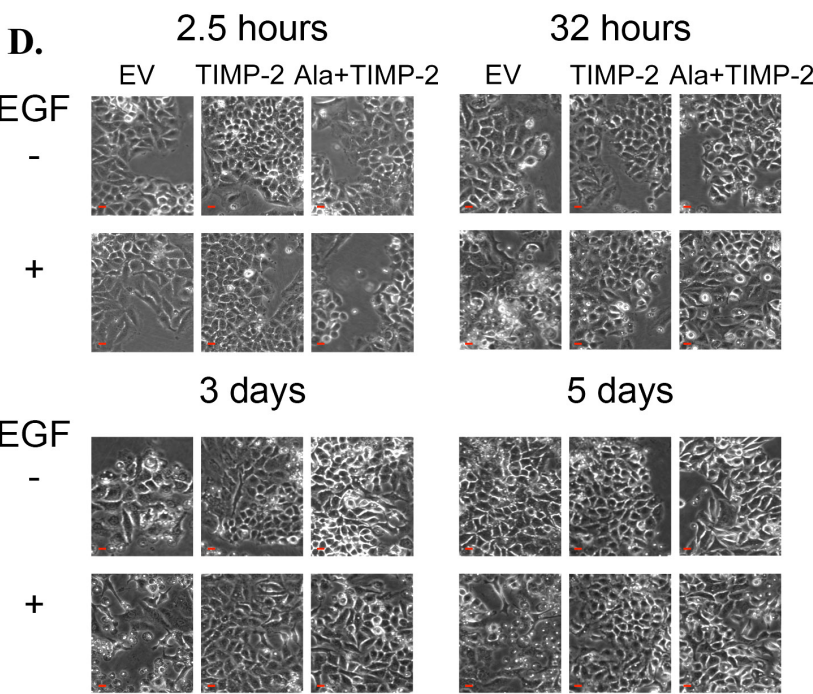

$2.5 \mathrm{hrs}$

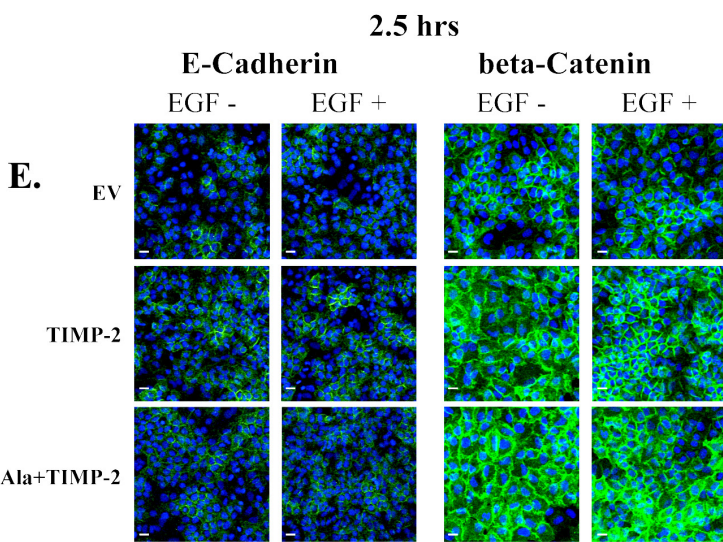

3 days E-Cadherin beta-Catenin

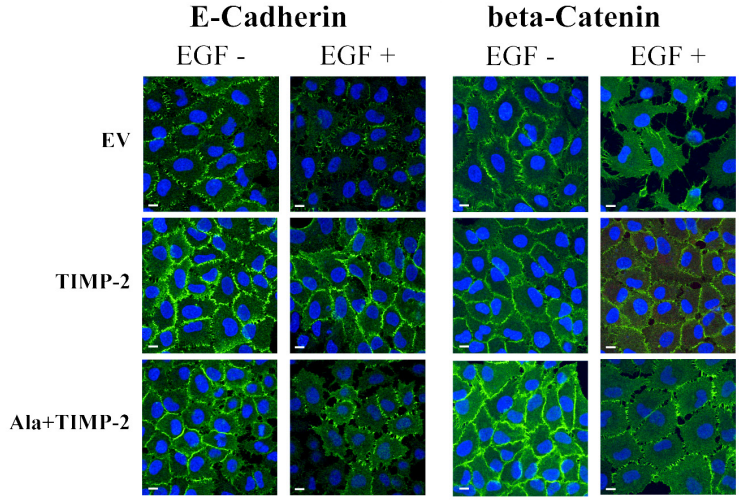

Figure 2: TIMP-2 overexpression upregulates E-cadherin and beta-catenin complex. (A) A cDNA microarray analysis heat map showing differentially expressed genes related to EMT in A549 EV, TIMP-2 and Ala+TIMP-2 in vitro (FDR $<5$ ). The key on the top left assigns heat map colors to the absolute gene expression value on a $\log _{2}$ scale. (B) Real time quantitative RT-PCR and (C) Western blot were performed in A549 cells on selected genes and proteins. (D) Time course experiment showing morphological changes in A549 transfected cells untreated or treated with EGF for $2.5 \mathrm{hrs}, 32 \mathrm{hrs}, 3$ days and 5 days (scale bars: $50 \mu \mathrm{m}$ ) and (E) immunofluorescence staining of E-cadherin and beta-catenin $2.5 \mathrm{hrs}$ and 3 days post EGF treatment. 


\section{TIMP-2 enhances E-cadherin expression and inhibits EGF-induced EMT}

One of the genes identified to be up-regulated in both TIMP-2 and Ala+TIMP-2 profiles is E-cadherin $(C D H 1)$, a gene expressed in most normal epithelial tissues. However, its loss in several carcinomas leads to increased tumor invasiveness, angiogenesis and epithelial to mesenchymal transition (EMT) [42, 43]. Therefore, we investigated the role of TIMP-2 in lung cancer development by analyzing genes associated with cell adhesion and EMT (Fig. 2A-C). Although some genes associated with mesenchymal phenotype also showed altered expression (CDH2, SERPINE1), E-cadherin was up-regulated in both TIMP-2 and Ala+TIMP-2 overexpressing A549 cells [44]. Since the C-terminus of E-cadherin binds directly to beta-catenin to stabilize cellcell adhesion, we also examined beta-catenin expression (Fig. 2B, C) [45]. Indeed, both E-cadherin and betacatenin were increased in TIMP-2 and Ala+TIMP-2 cells, both transcriptionally and at the protein level.

It has been previously demonstrated that treatment of A549 cells with EGF induces EMT via disruption of cell-cell adhesion, internalization of E-cadherin from cell-cell contacts, cellular morphological changes from cobblestone to spindle shape, and increased cell motility [46]. Here, treatment of A549 EV cells with EGF $(100 \mathrm{ng} / \mathrm{ml})$ for 2.5 hours, 32 hours, 3 days and 5 days resulted in disruption of cell-cell adhesion and contacts (spindle shaped, elongated cells), whereas TIMP-2 and Ala+TIMP-2 overexpressing cells were resistant to chronic EGF treatment (3 and 5 days) (Fig. 2D). Additionally, previous studies demonstrated that reduced E-cadherin expression correlates with tumor invasion, metastasis and poor prognosis in patients with lung cancer $[28,47]$. Here we show that both E-cadherin and betacatenin are strongly localized at the plasma membrane and remain so after exposure to EGF in A549 TIMP2 and Ala+TIMP-2, compared to EV cells (Fig. 2E). In contrast, EV cells treated with EGF showed reduced levels of both E-cadherin and beta-catenin expression at the plasma membrane and disrupted cell-cell adhesion. It was recently shown that TIMP-1 signaling promotes MDCK cell migration and invasion and induces an EMT program that also includes FAK activation [48]. TIMP-1, as opposed to TIMP-2, is overexpressed in a number of cancers, promoting tumor growth and inhibiting apoptosis, suggesting that the functional properties of TIMP family members are distinct. Indeed, we have previously shown that forced expression of TIMP-2 or Ala+TIMP-2 in A549 cells decreases both AKT and FAK phosphorylation in tumor xenografts [12].
Exogenous treatment with Ala+TIMP-2 upregulates E-cadherin in A549 cells and a linear correlation exists between TIMP-2 and E-cadherin mRNA levels in NSCLC cell lines.

Since stable transfection may alter cellular functionality, we addressed whether exogenous treatment of wild type A549 cells with Ala+TIMP-2 prior to addition of EGF would affect expression and localization of E-cadherin and beta-catenin (Fig. 3A). EGF treatment for $2 \mathrm{hrs}$ decreased the levels of both beta-catenin and E-cadherin from the plasma membrane. However, Ala+TIMP-2 pretreatment for $10 \mathrm{~min}$ induced upregulation of both E-cadherin and beta-catenin and, more importantly, prevented EGF induced down-regulation of E-cadherin and beta-catenin from the plasma membrane.

We then examined whether our findings are specific for A549 cells or whether other lung cancer cell lines show a similar pattern. We selected four non-small cell lung cancer (NSCLC) cell lines, H23, H460, A549 and $\mathrm{H} 358 \mathrm{M}$, and we determined the endogenous levels of both E-cadherin and TIMP-2 by real time PCR (Fig. 3B). Our results indicate that there is a linear co-relation between TIMP-2 and E-cadherin mRNA levels in these cells $\left(p=0.0439, r^{2}=0.9141\right)$, indicating that the expression of both molecules may be modulated by similar mechanisms.

\section{A549 xenografts show increased E-cadherin protein expression and display a transcriptional profile related to decreased tumor cell growth and metastasis}

TIMP-2 and Ala+TIMP-2 A549 xenografts exhibited reduced tumor growth (70-95\% inhibition), decreased angiogenesis and increased apoptosis in both nude and NOD-SCID mice (for tumor volumes and growth see $[12,21])$. Immunohistochemical analysis of tumor tissue revealed increased E-cadherin expression in TIMP-2 and Ala+TIMP-2 xenografts (Fig. 3C). Previous data show that membrane loss of E-cadherin leads to cell-cell separation and promotes the angiogenic switch in lung cancer [43]. Taken together, our recent findings of decreased microvascular density in TIMP-2 and Ala+TIMP-2 xenografts indicate that novel additional mechanisms may contribute to the anti-angiogenic effects of TIMP-2 in vivo [12].

To understand the TIMP-2 effects on the tumor microenvironment and how these have contributed to the inhibition of tumor growth in vivo, we analyzed the transcriptional profiles of A549 TIMP-2 and Ala+TIMP-2 xenografts at day 21 post inoculation in NOD-SCID mice (Supplementary Fig 1B) [21]. TIMP-2 xenografts revealed several specific functions associated with decreased tumor metastasis and lipid metabolism, while 
A.

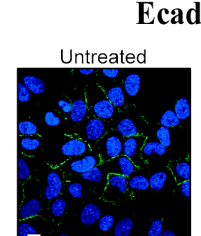

Ecadherin
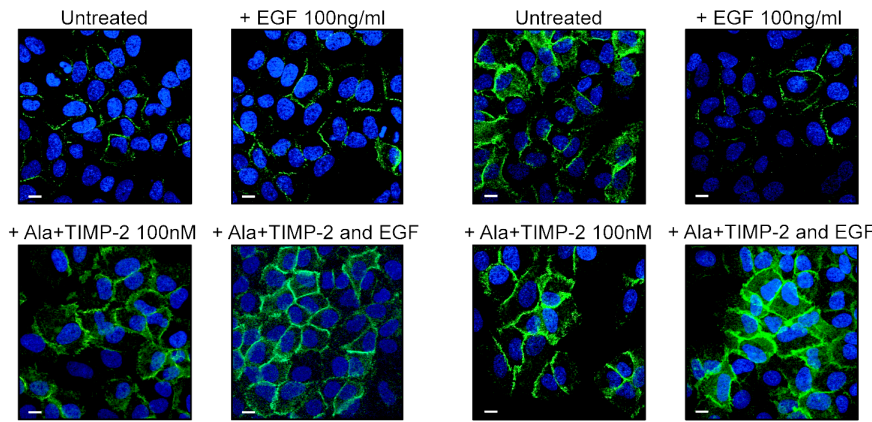

B.

Ecadherin and TIMP-2 mRNA levels

Relationship between endogenous TIMP-2 and E-cadherin mRNA levels
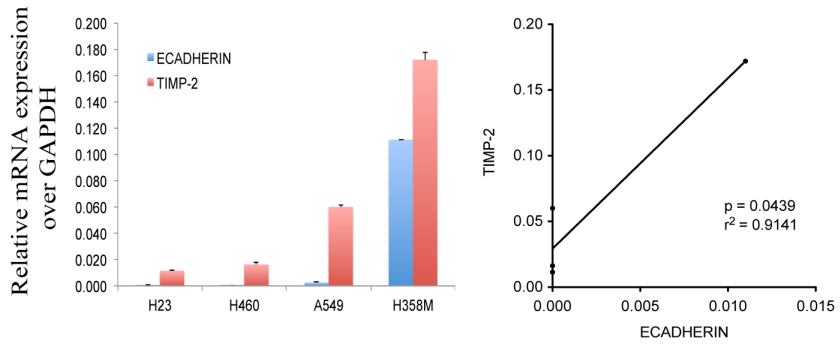

C.

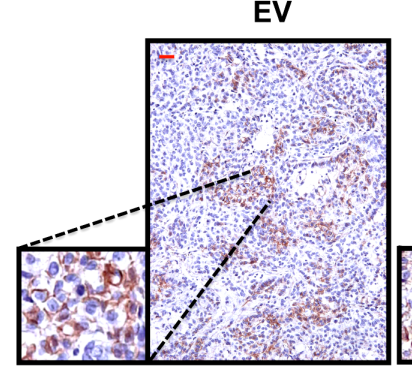

TIMP-2

Ala+TIMP-2

D.

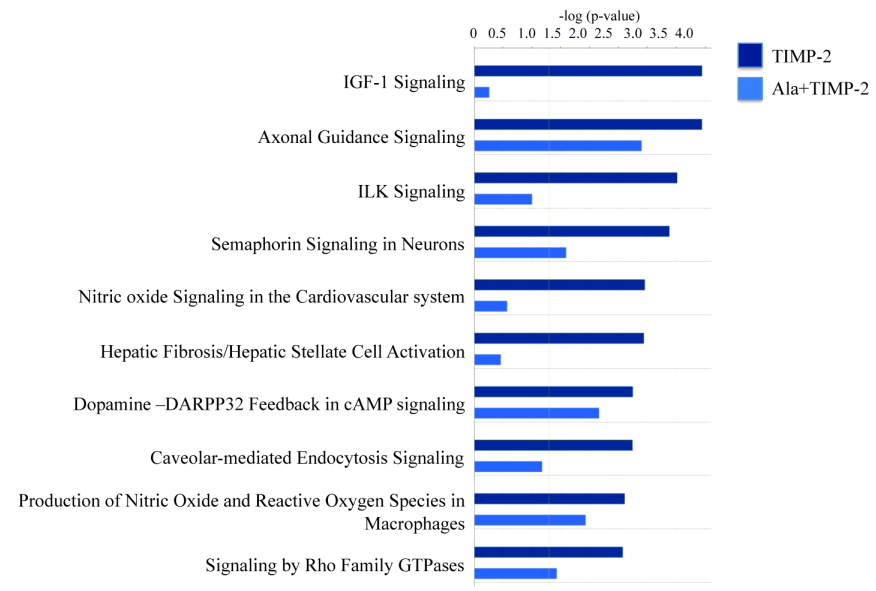

Figure 3: Exogenous treatment with Ala+TIMP-2, TIMP-2 expression in NCSLC and transcriptional analysis of tumor xenografts. (A) Exogenous treatment with 100nM Ala+TIMP-2 on $24 \mathrm{hr}$ serum starved A549 cells with and without additional treatment with $100 \mathrm{ng} / \mathrm{ml}$ EGF for $2 \mathrm{hrs}$. Cells were stained with either E-cadherin (left) or beta-catenin (right) (scale bars: $50 \mu \mathrm{m}$ ). (B) Endogenous TIMP-2 gene expression levels have linear correlation with endogenous E-cadherin expression levels in 4 non-small cell lung cancer cell lines (NSCLC). (LEFT) The data presented as means+SEM of three independent experiments. Real time qRT-PCR analysis for TIMP-2 and E-cadherin in 4 NSCLC (C) Expession of E-cadherin was determined by immunohistochemistry in A549 xenografts (scale bars: 50 $\mu \mathrm{m}$, inserts $200 \mu \mathrm{m}$ ). Each photograph represents screening of 20 high power fields (at 100x magnification). (D) Comparisons between A549 TIMP-2 or Ala+TIMP-2 with EV were performed and a list of differentially expressed genes from each was taken for further study on Ingenuity Pathway Analysis (IPA) to identify enriched pathways. Top 10 canonical pathways activated by TIMP-2 and/or Ala+TIMP-2 are shown with a threshold value of $<0.05$ (yellow line). 
Ala+TIMP-2 xenografts showed profiles associated with decreased tumor growth. In an attempt to explain the tumor growth inhibition in the TIMP-2 and Ala+TIMP-2 xenografts, canonical signaling pathways were analyzed (Fig. 3D). The most significant pathways, as determined by Ingenuity Pathway Analysis (IPA), included insulinlike growth factor-1 (IGF-1) signaling, axonal guidance signaling, integrin linked kinase (ILK) signaling, and semaphorin signaling in neurons (Fig. 3D). As shown, the IGF-1 canonical pathway, a network that regulates cellular proliferation and apoptosis in several cancers and is implicated in increased lung cancer risk, was negatively affected by TIMP-2 overexpression (Fig. 3D) [49, 50]. More specifically, when compared to EV tumors, TIMP-2 or Ala+TIMP-2 caused down-regulation of IGF-1 receptor (IGF-1R) expression (4.8 fold) and an increase in IGF binding proteins (including IGFBP-5 (4.0 fold) and -7 (3.5 fold)), proteins that limit the bioavailability of IGF ligands [51]. Given that inhibition of IGF-1R signaling leads to increased chemosensitivity in A549 cells, manipulation of the IGF signaling pathway may provide a useful therapeutic target for cancer therapy [52-55]. TIMP-2 has also been shown to inhibit IGF-1R directly by binding to the receptor expressed on endothelial cells $[10,11]$. We, therefore, conclude that TIMP-2 acts on tumor cells and the tumor microenvironment to inhibit tumor growth.

We, and others, have previously described MMP dependent and independent anti-angiogenic properties of

$$
\begin{array}{ll}
\longrightarrow=\text { Inhibits } & \mathbf{4}=\text { upregulation } \\
\longrightarrow=\text { Promotes/Activates } & \downarrow=\text { downregulation } \\
\boldsymbol{X}=\text { =binding } & \square=\text { previous studies }
\end{array}
$$

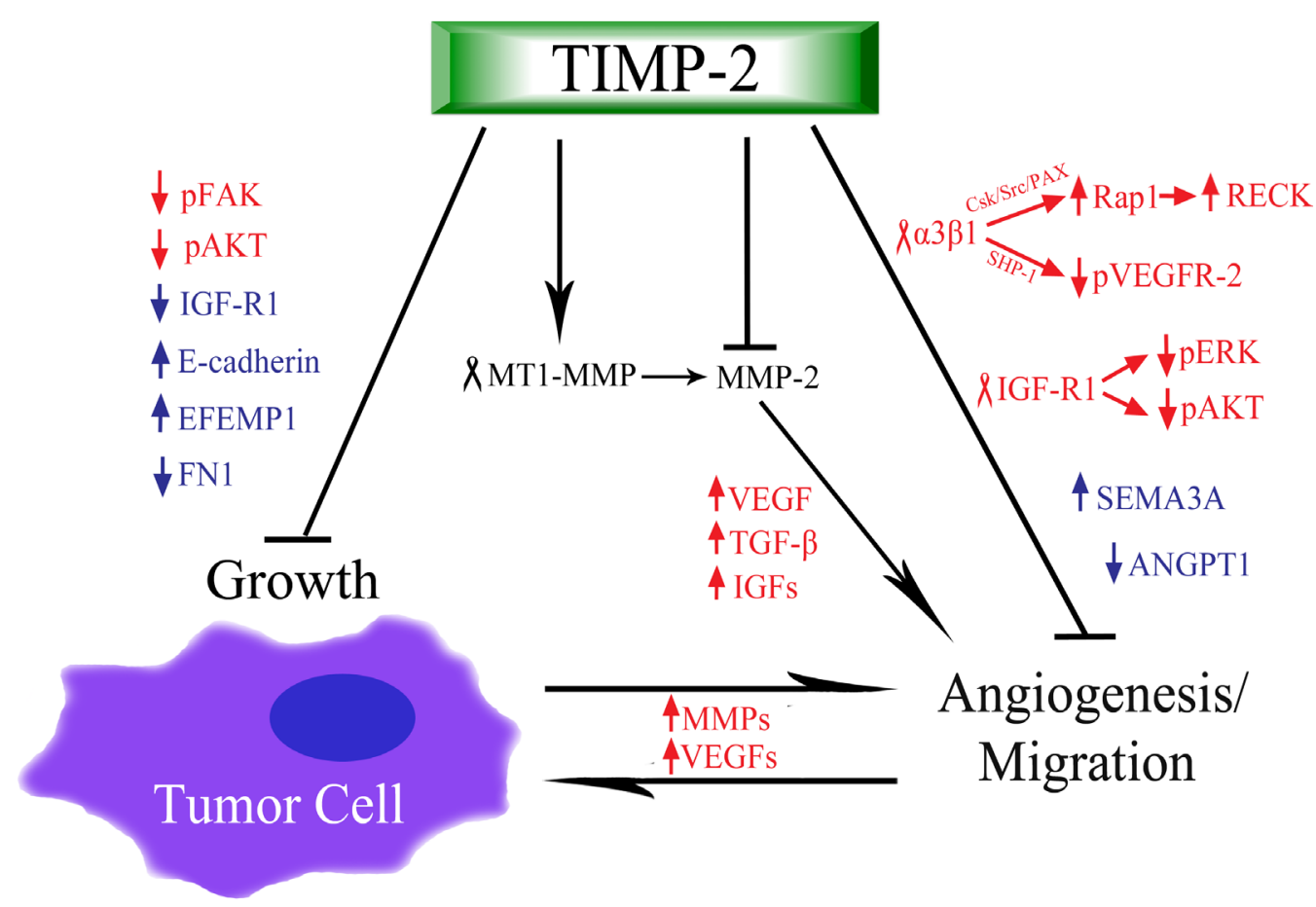

Figure 4: Schematic summary of TIMP-2 anti-tumoral and anti-angiogenic functions. TIMP-2 is primarily understood to both inhibit and activate (via MMP-14/MT1-MMP) MMP-2, which breaks down the ECM, releasing various growth factors (e.g. VEGFs, TGF- $\beta$, IGFs) that promote angiogenesis and cell proliferation [1-5]. The process of angiogenesis further induces the up-regulation of MMPs and VEGFs, thus, creating a positive feedback that furthers vascular development and cell growth. Independent of MMP inhibition and activation, TIMP-2 binds to integrin $\alpha_{3} \beta_{1}$ receptor, which causes signaling cascades (moderated by SHP-1 and Csk/Src/ PAX/Rap1, respectively) that lead to the hypo-phosphorylation of VEGFR-2 and the up-regulation of the anti-migration factor, RECK [12, 20, 41]. Also, previous studies have shown that TIMP-2 binds to endothelial IGF-R1, which causes a net reduction in the phosphorylation of ERK and AKT, and the subsequent decrease in endothelial growth and angiogenesis [11]. Additional TIMP-2 induced, decreases in angiogenic function include the up-regulation of semaphorin-3A and the down-regulation of angiopoietin-1 (Fig 1C). Furthermore, TIMP2 is associated with the inhibition of tumor, which has been paired with the observed hypo-phosphorylation of FAK and AKT, proteins that contribute to the proliferation and growth of tumor cells $[12,13]$. Additionally, as presented in this study, TIMP-2 induces the upregulation of E-cadherin, CDH1, which increases cell-cell adhesion, effectively inhibiting tumor cell growth, migration, and epithelial to mesenchymal transition (EMT). Finally, additional signs of TIMP-2 induced inhibition of tumor cell growth are the up-regulation of EFEMP1 and the down-regulation of fibronectin-1 (Fig 1B). 
TIMP-2 (Fig. 4). TIMP-2 can inhibit MMP-2 and overall migration and invasion of tumor endothelium. In addition, TIMP-2 inhibits blood vessel formation via receptor mediated mechanisms, including binding to integrin $\alpha_{3} \beta_{1}$ on endothelial cells and inactivating receptor tyrosine kinases including VEGFR-2 and FGFR-2 through dephosphorylation mediated by SHP-1. Additionally, TIMP2 may also bind to IGFR-1 and decrease downstream signaling. In the current study, we have identified a novel anti-tumor activity of TIMP-2 - namely an ability to modify the transcriptional profile of tumor cells, resulting in increased E-cadherin expression and down-regulation of IGFR-1 signaling components. Decreased tumorigenesis and metastasis could be associated with increased E-cadherin expression in tumor cells, while inhibition of tumor angiogenesis may involve down-regulation of the IGFR-1 signaling pathway as well as tumor derived antiangiogenic signals. Our study describes the anti-tumoral transcriptional signature of TIMP-2 in lung cancer cells.

\section{METHODS}

\section{Cell culture and cell culture conditions}

A549 cell culture, retroviral transfection and analysis were performed and comprehensive characterization and confirmation of inability of Ala+TIMP-2 to inhibit MMP has been previously shown [12]. A549 EV, TIMP-2 and Ala+TIMP-2 were grown to $80 \%$ confluency prior to replacing with fresh medium containing $0.5 \%$ fetal calf serum for 24 hours to induce quiescence. Cells were treated with or without EGF (100ng/ml) (BD Biosciences, San Jose, CA) for the indicated times (2.5 hours, 32 hours, 3 days and 5 days) and morphological changes were observed by phase-contrast microscopy (Olympus 1X70, Center Valley, PA). Exogenous treatment with Ala+TIMP-2 (100nM) was performed on 24-hours serum starved cells for $30 \mathrm{~min}$. EGF was added at $100 \mathrm{ng} / \mathrm{ml}$ for 2 hours.

\section{Cell line, tumor RNA isolation and real time quantitative RT-PCR}

Total RNA from three independent exponential growth phase cell cultures of A549 EV, TIMP-2, Ala+TIMP-2 and the 4 NSCLC cell lines was isolated using the RNAeasy kit (Qiagen, Gaithersburg, MD). Reverse transcription was performed as previously described [12]. Xenograft tumors were homogenized using QIAshredder (Invitrogen, Grand Island, NY) and RNA purified using the RNAeasy kit according to the manufacturer's instruction. Equal amounts of RNA (500ng) from six tumors per group were pooled into one sample and used to perform microarray analysis in tumor xenografts. TIMP-2, GAPDH primer sequences, real-time PCR conditions and analysis of gene expression were given elsewhere [12]. $500 \mathrm{nmol} / \mathrm{L}$ forward and reverse primers for E-cadherin and beta-catenin were used. Primer sequences for E-cadherin: forward, 5'GGTCAGCGTGTGTGACTGTG -3 ' and reverse, 5'- GCAGAATCAGAATTAGCAAAGCAAG -3' and Beta-catenin: 5'- GCAGAAAATGGTTGCCTTGCT-3' and reverse, 5' - GCAGAAAATGGTTGCCTTGCT -3'. Mean CT values for target genes were normalized for the endogenous control GAPDH. The ratio of mRNA expression of target gene versus $G A P D H$ was defined as $2(-\Delta \mathrm{Ct})$. All data are shown as mean \pm S.E.M. of at least three independent experiments.

\section{Western blot analysis}

Immunoblotting for TIMP-2 was performed in conditioned media (CM) as previously described [12]. Cell lysates were collected for analysis of E-cadherin and beta-catenin protein levels and probed overnight with mouse anti-E-cadherin antibody, 1/500 dilution (Abcam, Cambridge, MA) and mouse anti-beta-catenin antibody, 1/12,000 (BD Transduction, San Jose, CA).

\section{Immunofluorescence and Immunohistochemistry}

For immunofluorescence studies, A549 cells were cultured on 4-well glass slides (NUNC, Rochester, NY), serum starved, and treated with EGF $(100 \mathrm{ng} / \mathrm{ml})$ for 2.5 or 32 hours. Prior to staining, cells were fixed in $4 \%$ paraformaldehyde (Sigma) for $10 \mathrm{~min}$, permeabilized in $0.1 \%$ Triton X-100 for $20 \mathrm{~min}$, and blocked overnight in $1 \% \mathrm{BSA}$ at $4^{\circ} \mathrm{C}$ with two washes in PBS at room temperature between each of the previous steps. Cells were incubated with mouse monoclonal antibodies to E-Cadherin (Abcam, 1:100) or Beta-catenin (Cell Signaling, 1:500) overnight at $4^{\circ} \mathrm{C}$ in a humidity chamber followed by incubation with goat anti mouse Alexa-488 secondary antibody (Cell Signaling, 1:800) for 1 hour at room temperature. Both primary and secondary antibodies were diluted in Antibody Diluent Solution (Dako, Carpenteria, CA). Representative images were captured using the Zeiss LSM510 Meta Confocal Microscope. The E-cadherin expression in resected tumors was determined by immunohistochemistry as previously described [12].

\section{Microarray analysis in A549 cell lines and xenograft tumors}

Triplicate samples from A549 tumor cell lines representing 3 groups: EV 'control', TIMP-2 and Ala+TIMP-2 overexpressing cells were hybridized against the probes on Affymetrix HGU-133 Plus 2.0 chips. The 
array data were processed using the R/Bioconductor Statistical Package. MAS 5.0 normalization was performed on the probes and those with missing values were filtered. 20729 probes ( $s d \geq 0.2$ ) were retained for further analysis. Principal component analysis (PCA) and unbiased clustering on probe subsets were used to test the integrity of the arrays (data not shown). The 'two-class unpaired' t-test was performed using Significance of Microarray (SAM) module on R between samples TIMP2 and EV, or Ala+TIMP-2 and EV individually to derive differentially expressed genes. 'Multiclass' ANOVA was performed using SAM module to extract significantly affected probes across all three sample groups. False Discovery Rate (FDR) cut-off (10\%) was used to generate the lists of differentially expressed (DE) genes.

Single microarray samples (see above, tumor RNA isolation) derived using xenografts from EV, TIMP-2 and Ala+TIMP-2 tumor cell lines were processed and normalized using protocol described above. A fold change analysis was performed between the three sample classes.

DE gene lists from SAM and fold change analyses were used to extract enriched pathways on Ingenuity Pathway Analysis (IPA) software. An unbiased hierarchical clustering on the median centered and normalized DE genes was performed on Cluster version 3.0 and visualized on Java TreeView version 1.16r2.

\section{A549 Xenografts and tumor processing.}

The inoculation of A549 EV, TIMP-2 and Ala+TIMP-2 cells into NOD-SCID mice ( $n=15$ per group) and growth curves are described elsewhere [12, 21]. The mice were sacrificed 21 days post injection, and tumors were harvested. Necrotic tissue was removed, and the tumors were either processed immediately or stored in RNAlater (Ambion, Grand Island, NY) for RNA extraction.

\section{ACKNOWLEDGMENTS}

We thank the Laboratory of Molecular Technology (NCI Frederic) for performing the microarray experiment and the members of the Extracellular Matrix Pathology Section, Radiation Oncology Branch, NCI for helpful suggestions and to their contributions to the research reviewed in this manuscript. We thank Dr. Mehdi Mollapour, Urologic Oncology Branch, NCI for his helpful discussions.

\section{Funding}

This work was funded by the NCI Center for Cancer Research Intramural Research Program Grant \# ZIA SC009179-22.

\section{REFERENCE}

1. Stetler-Stevenson WG and Yu AE. Proteases in invasion: matrix metalloproteinases. Semin Cancer Biol. 2001; 11(2):143-152.

2. Nguyen DX, Bos PD and Massague J. Metastasis: from dissemination to organ-specific colonization. Nat Rev Cancer. 2009; 9(4):274-284.

3. Kessenbrock K, Plaks V and Werb Z. Matrix metalloproteinases: regulators of the tumor microenvironment. Cell. 2010; 141(1):52-67.

4. Hernandez-Barrantes S, Bernardo M, Toth M and Fridman R. Regulation of membrane type-matrix metalloproteinases. Semin Cancer Biol. 2002; 12(2):131-138.

5. Strongin AY, Collier I, Bannikov G, Marmer BL, Grant GA and Goldberg GI. Mechanism of cell surface activation of 72-kDa type IV collagenase. Isolation of the activated form of the membrane metalloprotease. J Biol Chem. 1995; 270(10):5331-5338

6. Albini A, Melchiori A, Santi L, Liotta LA, Brown PD and Stetler-Stevenson WG. Tumor cell invasion inhibited by TIMP-2. J Natl Cancer Inst. 1991; 83(11):775-779.

7. Imren S, Kohn DB, Shimada H, Blavier L and DeClerck YA. Overexpression of tissue inhibitor of metalloproteinases-2 retroviral-mediated gene transfer in vivo inhibits tumor growth and invasion. Cancer Res. 1996; 56(13):2891-2895.

8. DeClerck YA, Perez N, Shimada H, Boone TC, Langley $\mathrm{KE}$ and Taylor SM. Inhibition of invasion and metastasis in cells transfected with an inhibitor of metalloproteinases. Cancer Res. 1992; 52(3):701-708.

9. Seo DW, Li H, Guedez L, Wingfield PT, Diaz T, Salloum R, Wei BY and Stetler-Stevenson WG. TIMP-2 mediated inhibition of angiogenesis: an MMP-independent mechanism. Cell. 2003; 114(2):171-180.

10. Fernandez CA, Butterfield C, Jackson G and Moses MA. Structural and functional uncoupling of the enzymatic and angiogenic inhibitory activities of tissue inhibitor of metalloproteinase-2 (TIMP-2): loop 6 is a novel angiogenesis inhibitor. J Biol Chem. 2003; 278(42):4098940995.

11. Fernandez CA, Roy R, Lee S, Yang J, Panigrahy D, Van Vliet KJ and Moses MA. The anti-angiogenic peptide, loop 6 , binds insulin-like growth factor-1 receptor. J Biol Chem. 2010; 285(53):41886-41895.

12. Bourboulia D, Jensen-Taubman S, Rittler MR, Han HY, Chatterjee T, Wei B and Stetler-Stevenson WG. Endogenous Angiogenesis Inhibitor Blocks Tumor Growth via Direct and Indirect Effects on Tumor Microenvironment. Am J Pathol. 2011; 179(5):2589-2600.

13. Stetler-Stevenson WG, Bourboulia D, Guedez L and Jensen-Taubman S. Anti-tumoral Properties of Endogenous Angiogenesis Inhibitors: A case for Continued TIMP-2 Preclinical Development. Current Angiogenesis. 2012; 
1(2):148-156.

14. Lluri G, Langlois GD, McClellan B, Soloway PD and Jaworski DM. Tissue inhibitor of metalloproteinase-2 (TIMP-2) regulates neuromuscular junction development via a beta1 integrin-mediated mechanism. J Neurobiol. 2006; 66(12):1365-1377.

15. Lluri G, Langlois GD, Soloway PD and Jaworski DM. Tissue inhibitor of metalloproteinase-2 (TIMP-2) regulates myogenesis and beta1 integrin expression in vitro. Exp Cell Res. 2008; 314(1):11-24.

16. Oh J, Diaz T, Wei B, Chang H, Noda M and StetlerStevenson WG. TIMP-2 upregulates RECK expression via dephosphorylation of paxillin tyrosine residues 31 and 118 . Oncogene. 2006; 25(30):4230-4234.

17. Oh J, Seo DW, Diaz T, Wei B, Ward Y, Ray JM, Morioka Y, Shi S, Kitayama H, Takahashi C, Noda M and StetlerStevenson WG. Tissue inhibitors of metalloproteinase 2 inhibits endothelial cell migration through increased expression of RECK. Cancer Res. 2004; 64(24):9062-9069.

18. Perez-Martinez L and Jaworski DM. Tissue inhibitor of metalloproteinase-2 promotes neuronal differentiation by acting as an anti-mitogenic signal. J Neurosci. 2005; 25(20):4917-4929.

19. Wingfield PT, Sax JK, Stahl SJ, Kaufman J, Palmer I, Chung V, Corcoran ML, Kleiner DE and Stetler-Stevenson WG. Biophysical and functional characterization of full-length, recombinant human tissue inhibitor of metalloproteinases-2 (TIMP-2) produced in Escherichia coli. Comparison of wild type and amino-terminal alanine appended variant with implications for the mechanism of TIMP functions. J Biol Chem. 1999; 274(30):21362-21368.

20. Bourboulia D and Stetler-Stevenson WG. Matrix metalloproteinases (MMPs) and tissue inhibitors of metalloproteinases (TIMPs): Positive and negative regulators in tumor cell adhesion. Semin Cancer Biol. 2010; 20(3):161-168.

21. Bourboulia D, Jensen-Taubman S and Stetler-Stevenson WG. TIMP-2: An Endogenous Angiogenesis Inhibitor with Distinch Antitumoral Properties. Treatment Strategies Hematology. 2012; 2(2):31-35.

22. Hu Y, Pioli PD, Siegel E, Zhang Q, Nelson J, Chaturbedi A, Mathews MS, Ro DI, Alkafeef S, Hsu N, Hamamura M, Yu L, Hess KR, Tromberg BJ, Linskey ME and Zhou YH. EFEMP1 suppresses malignant glioma growth and exerts its action within the tumor extracellular compartment. Mol Cancer. 2011; 10:123.

23. Durkin ME, Yuan BZ, Zhou X, Zimonjic DB, Lowy DR, Thorgeirsson SS and Popescu NC. DLC-1:a Rho GTPaseactivating protein and tumour suppressor. J Cell Mol Med. 2007; 11(5):1185-1207.

24. Strickland LA, Ross J, Williams S, Ross S, Romero M, Spencer S, Erickson R, Sutcliffe J, Verbeke C, Polakis P, van Bruggen $\mathrm{N}$ and Koeppen $\mathrm{H}$. Preclinical evaluation of carcinoembryonic cell adhesion molecule (CEACAM) 6 as potential therapy target for pancreatic adenocarcinoma. J Pathol. 2009; 218(3):380-390.

25. Carraway RE and Plona AM. Involvement of neurotensin in cancer growth: evidence, mechanisms and development of diagnostic tools. Peptides. 2006; 27(10):2445-2460.

26. Cao H, Wang G, Meng L, Shen H, Feng Z, Liu Q and Du J. Association between Circulating Levels of IGF-1 and IGFBP-3 and Lung Cancer Risk: A Meta-Analysis. PLoS One. 2012; 7(11):e49884.

27. Bremnes RM, Veve R, Gabrielson E, Hirsch FR, Baron A, Bemis L, Gemmill RM, Drabkin HA and Franklin WA. High-throughput tissue microarray analysis used to evaluate biology and prognostic significance of the E-cadherin pathway in non-small-cell lung cancer. J Clin Oncol. 2002; 20(10):2417-2428.

28. Bremnes RM, Veve R, Hirsch FR and Franklin WA. The E-cadherin cell-cell adhesion complex and lung cancer invasion, metastasis, and prognosis. Lung Cancer. 2002; 36(2):115-124.

29. Rehbein G, Simm A, Hofmann HS, Silber RE and Bartling B. Molecular regulation of S100P in human lung adenocarcinomas. Int J Mol Med. 2008; 22(1):69-77.

30. Hida T, Yatabe Y, Achiwa H, Muramatsu H, Kozaki K, Nakamura S, Ogawa M, Mitsudomi T, Sugiura T and Takahashi $T$. Increased expression of cyclooxygenase 2 occurs frequently in human lung cancers, specifically in adenocarcinomas. Cancer Res. 1998; 58(17):3761-3764.

31. Lin MI, Yu J, Murata T and Sessa WC. Caveolin-1-deficient mice have increased tumor microvascular permeability, angiogenesis, and growth. Cancer Res. 2007; 67(6):28492856.

32. Assadian S, El-Assaad W, Wang XQ, Gannon PO, Barres V, Latour M, Mes-Masson AM, Saad F, Sado Y, Dostie $\mathrm{J}$ and Teodoro JG. p53 inhibits angiogenesis by inducing the production of Arresten. Cancer Res. 2012; 72(5):12701279.

33. Acevedo LM, Barillas S, Weis SM, Gothert JR and Cheresh DA. Semaphorin 3A suppresses VEGF-mediated angiogenesis yet acts as a vascular permeability factor. Blood. 2008; 111(5):2674-2680.

34. Gerstel D, Wegwitz F, Jannasch K, Ludewig P, Scheike K, Alves F, Beauchemin N, Deppert W, Wagener C and Horst AK. CEACAM1 creates a pro-angiogenic tumor microenvironment that supports tumor vessel maturation. Oncogene. 2011; 30(41):4275-4288.

35. Wang D and DuBois RN. Cyclooxygenase 2-derived prostaglandin E2 regulates the angiogenic switch. Proc Natl Acad Sci U S A. 2004; 101(2):415-416.

36. Isogai C, Laug WE, Shimada H, Declerck PJ, Stins MF, Durden DL, Erdreich-Epstein A and DeClerck YA. Plasminogen activator inhibitor-1 promotes angiogenesis by stimulating endothelial cell migration toward fibronectin. Cancer Res. 2001; 61(14):5587-5594.

37. Tamagnone L. Emerging role of semaphorins as major 
regulatory signals and potential therapeutic targets in cancer. Cancer Cell. 2012; 22(2):145-152.

38. Moore CS and Crocker SJ. An Alternate Perspective on the Roles of TIMPs and MMPs in Pathology. Am J Pathol. 2012; 180(1):12-16.

39. Walsh LA, Cepeda MA and Damjanovski S. Analysis of the MMP-dependent and independent functions of tissue inhibitor of metalloproteinase- 2 on the invasiveness of breast cancer cells. J Cell Commun Signal. 2012.

40. Murphy AN, Unsworth EJ and Stetler-Stevenson WG. Tissue inhibitor of metalloproteinases-2 inhibits bFGFinduced human microvascular endothelial cell proliferation. J Cell Physiol. 1993; 157(2):351-358.

41. Stetler-Stevenson WG. Tissue inhibitors of metalloproteinases in cell signaling: metalloproteinaseindependent biological activities. Sci Signal. 2008; 1(27):re6.

42. Ishiyama N, Lee SH, Liu S, Li GY, Smith MJ, Reichardt LF and Ikura M. Dynamic and static interactions between p120 catenin and E-cadherin regulate the stability of cellcell adhesion. Cell. 2010; 141(1):117-128.

43. Ceteci F, Ceteci S, Karreman C, Kramer BW, Asan E, Gotz $\mathrm{R}$ and Rapp UR. Disruption of tumor cell adhesion promotes angiogenic switch and progression to micrometastasis in RAF-driven murine lung cancer. Cancer Cell. 2007; 12(2):145-159.

44. Giehl $\mathrm{K}$ and Menke A. Microenvironmental regulation of E-cadherin-mediated adherens junctions. Front Biosci. 2008; 13:3975-3985

45. Kase S, Sugio K, Yamazaki K, Okamoto T, Yano T and Sugimachi K. Expression of E-cadherin and beta-catenin in human non-small cell lung cancer and the clinical significance. Clin Cancer Res. 2000; 6(12):4789-4796.

46. Lu Z, Ghosh S, Wang Z and Hunter T. Downregulation of caveolin-1 function by EGF leads to the loss of E-cadherin, increased transcriptional activity of beta-catenin, and enhanced tumor cell invasion. Cancer Cell. 2003; 4(6):499515.

47. Guilford P. E-cadherin downregulation in cancer: fuel on the fire? Mol Med Today. 1999; 5(4):172-177.

48. Jung YS, Liu XW, Chirco R, Warner RB, Fridman R and Kim HR. TIMP-1 induces an EMT-like phenotypic conversion in MDCK cells independent of its MMPinhibitory domain. PLoS One. 2012; 7(6):e38773.

49. Karamouzis MV and Papavassiliou AG. The IGF-1 network in lung carcinoma therapeutics. Trends Mol Med. 2006; 12(12):595-602.

50. Tang H, Liao Y, Chen G, Xu L, Zhang C, Ju S and Zhou S. Estrogen upregulates the IGF-1 signaling pathway in lung cancer through estrogen receptor-beta. Med Oncol. 2012.

51. Pollak M. Insulin and insulin-like growth factor signalling in neoplasia. Nat Rev Cancer. 2008; 8(12):915-928.

52. Badzio A, Wynes MW, Dziadziuszko R, Merrick DT, Pardo M, Rzyman W, Kowalczyk A, Singh S, Ranger-Moore J,
Manriquez G, Gaire F, Jassem J and Hirsch FR. Increased insulin-like growth factor 1 receptor protein expression and gene copy number in small cell lung cancer. J Thorac Oncol. 2010; 5(12):1905-1911.

53. Dong A, Kong M, Ma Z, Qian J, Cheng $\mathrm{H}$ and Xu X. Knockdown of insulin-like growth factor 1 receptor enhances chemosensitivity to cisplatin in human lung adenocarcinoma A549 cells. Acta Biochim Biophys Sin (Shanghai). 2008; 40(6):497-504.

54. Dziadziuszko R, Merrick DT, Witta SE, Mendoza AD, Szostakiewicz B, Szymanowska A, Rzyman W, Dziadziuszko K, Jassem J, Bunn PA, Jr., Varella-Garcia $\mathrm{M}$ and Hirsch FR. Insulin-like growth factor receptor 1 (IGF1R) gene copy number is associated with survival in operable non-small-cell lung cancer: a comparison between IGF1R fluorescent in situ hybridization, protein expression, and mRNA expression. J Clin Oncol. 2010; 28(13):21742180.

55. Pollak M. Targeting insulin and insulin-like growth factor signalling in oncology. Curr Opin Pharmacol. 2008; 8(4):384-392. 